\title{
The outermost capsular arabinomannans and other mannoconjugates of virulent and avirulent tubercle bacilli
}

\author{
Annick Ortalo-Magné,' Åse B. Andersen ${ }^{2}$ and Mamadou Daffé ${ }^{1}$ \\ Author for correspondence: Mamadou Daffé. Tel: +33613359 16. Fax: + 3361335886. \\ e-mail: daffe@1ptf.biotoul.fr
}

1 Laboratoire de

Pharmacologie et de Toxicologie

Fondamentales du CNRS

and Université Paul

Sabatier, 118 route de

Narbonne, 31062 Toulouse

Cedex, France

2 Mycobacteria Department, Statens Seruminstitut, 5 Artillerivej, DK-2300

Copenhagen S, Denmark

\begin{abstract}
It has been shown that phagocyte mannose receptors play an important role in phagocytosis of virulent tubercle bacilli, but not of avirulent strains.

Accordingly, we investigated the occurrence and structure of the outermost mannoconjugates of the capsule of five strains of the tubercle bacillus differing in their degrees of virulence. The extracellular and surface-exposed arabinomannan-containing polysaccharides were chemically characterized as being composed mainly of neutral fatty-acyl-free arabinomannans (AMs) possessing a reducing end consisting of mannose. Although no lipoarabinomannan (LAM) was detected, small amounts of acidic polysaccharides, exhibiting the same electrophoretic mobility as LAM, were identified as succinylated AMs (two to three residues per molecule) lacking the phosphatidylinositol anchor of LAM. AMs from the different strains shared the same structural features, notably the capping of a large portion of the arabinan segments with mannosyl residues. However, no correlation was observed between either the percentage of capping or the amount of AMs and the degrees of virulence of the strains. The occurrence and amounts of other mannoconjugates (phosphatidylinositol mannosides and the mannoseassociated 19 and $38 \mathrm{kDa}$ lipoproteins) in the various tubercle bacilli were also examined. Although both classes of compounds were identified in all the examined strains, a correlation between the amounts of the glycoconjugates and the degrees of virulence of the strains could not be established. These data do not support the implication of these promising mannosylated molecules in the selective phagocytosis of virulent tubercle bacilli and indicate that the involvement of mannose receptors in phagocytosis of virulent $M$. tuberculosis needs to be re-investigated.
\end{abstract}

Keywords: tuberculosis, Mycobacterium, glycoconjugates, arabinomannan, capsule

\section{INTRODUCTION}

Tuberculosis remains a major health problem, claiming three million lives annually. The World Health Organization estimates that one-third of the world's population is, or has been, infected with the tubercle bacillus and is at risk of developing the disease (Kochi, 1991). The steadily declining incidence of tuberculosis in industrialized countries over several decades has been reversed due to the

Abbreviations: AMs, arabinomannans; LAMs, lipoarabinomannans; LMs, lipomannans; ManAMs, mannose-capped arabinomannans; ManLAMs, mannose-capped lipoarabinomannans; PIMs, phosphatidylinositol mannosides. emergence of drug-resistant strains and the coincidence of tuberculosis and HIV infection (Bloom \& Murray, 1992). Mycobacterium tuberculosis, the causative agent of tuberculosis, is a facultative intracellular pathogen capable of resisting destruction and multiplying inside mononuclear phagocytic cells. Little is known, however, about the mechanisms of its pathogenicity. The initial step in the host entry by the bacillus is phagocytosis. It has been shown that complement receptors CR1, CR 3 and CR 4 are involved in the phagocytosis of mycobacteria, including M. tuberculosis (Bermudez et al., 1991; Hetland \& Wiker, 1994; Hirsch et al., 1994; Schlesinger, 1993; Schlesinger \& Horwitz, 1990, 1991; Schlesinger et al., 1990; Stokes et al., 1993). In addition to these receptors, the mannose 
receptors on monocyte-derived macrophages have recently been shown to mediate phagocytosis of the virulent Erdman and H37Rv strains, but not of the avirulent H37Ra strain (Schlesinger, 1993). Based on the structural differences found between lipoarabinomannans (LAMs) synthesized by the virulent Erdman and H37Rv strains [mannose-capped LAM (ManLAM), Chatterjee et al., 1992b; Venisse et al., 1993] and an unidentified mycobacterium, once thought to be an attenuated strain of $M$. tuberculosis (AraLAM, Chatterjee et al., 1991), polystyrene microspheres coated with purified ManLAMs and lipomannans (LMs) have been used to demonstrate the crucial role of the mannosyl units at the non-reducing ends of ManLAMs in adherence of coated microspheres to monocyte-derived macrophages (Schlesinger et al., 1994). The mannan core, which consists of a linear $(1 \rightarrow 6)-\alpha-$ mannan backbone with one $\alpha$-mannosyl unit branched at some positions 6 (Chatterjee et al., 1992a), has been shown not to mediate detectable binding of microspheres to monocyte-derived macrophages (Schlesinger et al., 1994). Thus, the terminal linear $(1 \rightarrow 2)-\alpha$-mannosyl residues of surface-exposed glycoconjugates may be an element of the phagocytic process leading to survival and early growth of the virulent tubercle bacilli in macrophages. However, an important unproved point is the surface-location of LAMs, a requirement for their possible interaction with phagocyte mannose receptors. Although monoclonal antibodies directed against LAMs have been shown to react with the surface of the unidentified mycobacterium (Hunter \& Brennan, 1990), these antibodies are known to be directed against carbohydrate epitopes and not against the lipid moiety of LAMs (Chatterjee et al., 1992b; Daffé et al., 1990; Ortalo-Magné et al., 1995; Prinzis et al., 1994). Thus, the presence of LAMs on the cell surface remained to be established. Furthermore, recent studies demonstrated that LAMs of attenuated, avirulent and virulent tubercle bacilli are all mannose-capped (Khoo et al., 1995; Venisse et al., 1993), suggesting that the presence of ManLAMs does not explain the selective interaction of virulent tubercle bacilli with the mannose receptors of phagocytes. Consequently, further studies are clearly needed for defining the molecules occurring in virulent tubercle bacilli that interact with the mannose receptors.

We recently isolated the extracellular and surface-exposed constituents of virulent strains of $M$. tuberculosis; these appeared to indicate the presence of a capsule composed mainly of polysaccharides and proteins (Lemassu \& Daffé, 1994; Ortalo-Magné et al., 1995). The major capsular polysaccharides were a glycogen-like high-molecularmass glucan, an extensively mannose-capped arabinomannan (ManAM) structurally related to ManLAMs and a mannan. Among the capsular proteins of the tubercle bacillus, carbohydrate was found associated with the purified 19 and $38 \mathrm{kDa}$ lipoproteins (Young et al., 1992) which have been shown to bind concanavalin A (Espitia \& Mancilla, 1989; Garbe et al., 1993). Interestingly, digestion of these proteins with $\alpha$-D-mannosidase produced a reduction in their molecular mass, demonstrating the abundance of $\alpha$-mannosyl units in the two glycoproteins (Espitia et al., 1993, 1995) and suggesting that they may represent potential candidates for the interaction with the mannose receptor. In the present study, we compared the major outermost AMs and the mannoseassociated lipoproteins of the virulent Erdman and H37Rv strains, the attenuated R1Rv strain and the avirulent H37Ra and R1Ra strains (Steenken \& Gardner, 1946a, b).

\section{METHODS}

Strains and growth conditions. $M$. tuberculosis strains Erdman (ATCC 35801), H37Rv (ATCC 27294), H37Ra (ATCC 25177), R1Rv (ATCC 35818) and R1Ra (ATCC 35819) were grown on the synthetic Sauton's medium (Sauton, 1912) (100 ml per flask) as surface pellicles at $37^{\circ} \mathrm{C}$.

Isolation, purification and chemical characterization of AMs. Culture filtrates were recovered by filtration through a no. 111 Durieux filter paper, followed by filtration through $0 \cdot 2 \mu \mathrm{m}$ filter (Nalgene). Cells were harvested from surface pellicles that remained attached to the flasks when recovering the culture filtrates. Bacteria were gently shaken (1000 r.p.m.) for $1 \mathrm{~min}$ with $10 \mathrm{~g}$ glass beads ( $4 \mathrm{~mm}$ diameter) per $2 \mathrm{~g}$ wet cells, a method known not to affect the integrity of cells (Ortalo-Magné et al., 1995). Distilled water ( $50 \mathrm{ml}$ per flask) was then added and the bacterial suspensions were filtered through $0.2 \mu \mathrm{m}$ filter. These filtrates and the original culture filtrates were concentrated under vacuum to one-tenth of the original volume. AMs from the different tubercle bacilli materials were purified and chemically characterized as previously described by Lemassu \& Daffé (1994).

To define the chemical nature of the reducing end of AMs, samples (5-7 mg) of purified polysaccharides and standard sugars [arabinose (Ara), mannose (Man), $N$-acetylglucosamine, glucosamine, $N$-acetylgalactosamine and galactosamine] were treated with a mixture of ethyl $p$-aminobenzoate, cyanoborohydride (in glacial acetic acid and methanol) as described by Venisse et al. (1993). The UV-absorbing compounds were purified using a reverse-phase Sep-Pak C18. They were eluted in the water/methanol $(8: 2, \mathrm{v} / \mathrm{v})$ fraction, hydrolysed, methylated with diazomethane and analysed by HPLC on a reversephase (C18) column using water/methanol (7:3, v/v) as eluent. Samples of the different HPLC peaks were analysed by GC after trimethylsilylation.

Electrophoresis. Samples of dialysed crude materials and purified polysaccharides were analysed by SDS-PAGE. Proteins were analysed in a $10-20 \%(\mathrm{w} / \mathrm{v})$ gradient of SDSpolyacrylamide under reducing conditions. Polysaccharides were analysed in a $12.5 \%$ acrylamide cross-linked with $0.8 \%$ bisacrylamide. A stacking gel composed of $6 \%$ acrylamide was used. The gels were silver-stained using the Bio-Rad method. Authentic standards of LAM, LM and phosphatidylinositol mannosides (PIMs) were kindly supplied by T. Brando (LPTFCNRS, Toulouse, France).

Western blot analysis. In Western blot (immunoblot) experiments, the proteins separated by PAGE were transferred onto nitrocellulose sheets as previously described (Ortalo-Magné $e t$ al., 1995). The blots were incubated for $2 \mathrm{~h}$ at room temperature (or overnight at $4{ }^{\circ} \mathrm{C}$ ) with monoclonal antibodies (mAbs) directed against specific secreted proteins, HYT 6 (Andersen $e t$ al., 1986) and HBT 12 (Ljungqvist et al., 1988). Bound mAbs were detected by using horseradish-peroxidase-coupled rabbit anti-mouse immunoglobulins as described by Ortalo-Magné $e t$ al. (1995). 
Chemical analyses of lipids. Wet cells were saponified and the fatty acids were extracted, derivatized and analysed, as previously described by Daffé et al. (1983).

Phosphatidylinositol mannosides (PIMs) were analysed on aluminium-backed sheets coated with silicagel 60 (Merck) using chloroform/methanol/water $(65: 25: 4$, by vol.) as the developing solvent. The glycophospholipid nature of PIMs was established by spraying the plates with $0.2 \%$ anthrone in concentrated sulphuric acid followed by heating at $110^{\circ} \mathrm{C}$ (for the detection of glycolipids) and by the Dittmer-Lester (1964) reagent (for the detection of phospholipids).

\section{RESULTS}

\section{Purification and chemical analyses of AMs}

The amounts of polymers derived from ethanol precipitation of the unfractionated culture filtrates varied according to the batch and usually represented $15-20 \%$, but may reach up to $50 \%$, of the corresponding dried bacterial mass. There was, however, no correlation between the amounts of extracellular material and the virulence of the various strains examined. The surfaceexposed materials obtained after the gentle shaking of cells with glass beads (Ortalo-Magné et al., 1995) represented invariably $2-3 \%$ of the dried bacterial mass. The glycosyl composition of the extracellular and surfaceexposed materials from the various batches was very similar and consisted mainly of glucose (Glc, 70-75\%), Ara (15-20\%) and Man (10-15\%) (Lemassu \& Daffé, 1994; Ortalo-Magné et al., 1995). When a chloroform/ methanol/water partition was applied to the ethanol precipitates, the water-soluble polysaccharides contained mainly Man and Ara as sugar constituents in a molar ratio of $1: 1$. Small amounts of xylose $(2-5 \%)$ were also detected in the acid hydrolysis products of the aqueous phases derived from the mechanical treatment of cells. The acid hydrolysis of the interphases liberated only Glc and that of the organic phases liberated Glc, Man and inositol but no Ara. AMs from culture filtrates and those from surface-exposed materials of the various strains were isolated by fractionation of the water phases: in both cases, most of the polysaccharides (85-90\%) were eluted from the anion-exchange column with the buffer containing no $\mathrm{NaCl}$. The remaining materials were eluted using $0 \cdot 1 \mathrm{M} \mathrm{NaCl}$.

Chromatography of these neutral polysaccharides on columns of Bio-Gel P-10 calibrated using dextran standards led invariably to the isolation of AMs as the major peaks eluting in a position corresponding to a molecular mass of $13 \mathrm{kDa}$ (Lemassu \& Daffé, 1994). That the polysaccharides are not generated due to physical shearing or enzymic degradation (e.g. mannosidases) was supported by the isolation of AMs exhibiting similar chromatographic behaviour from the outermost cell surface components extracted from the bacilli with an overnight treatment of cells with Tween 80 . In addition, the expected heterogeneity in the size distribution of AMs if the polysaccharides derived from degradations was not observed.

No obvious relation was noted between the amounts of recovered AMs and the degrees of virulence of the bacilli (Table 1). For instance, comparable amounts of AMs were isolated and purified from the surface-exposed materials of both the virulent Erdman strain and the avirulent $\mathrm{H} 37 \mathrm{Ra}$ strain. The Ara/Man ratio in the purified AMs was invariable in the different batches of the same strain but may vary according to the origin of the material (extracellular or surface-exposed AMs) for a given strain (Table 1). The extracellular AMs from the Erdman and $\mathrm{H} 37 \mathrm{Rv}$ strains showed the lowest content of Man (Ara/Man ratio > 3). However, the Ara/Man ratio of the corresponding surface-exposed molecules from the same strains was similar to that found for the AMs isolated from the other strains (Ara/Man =1-2).

Analysis of the different purified neutral AMs by ${ }^{1} \mathrm{H}-$ NMR (Fig. 1a) showed that they were devoid of acyl substituent, as no signal was observed in the high field

Table 1. Glycosyl composition and amounts of AMs from various tubercle bacilli

AMs from the various extracellular materials (E) and surface-exposed materials (S) were obtained by gel filtration chromatography on columns of Bio-Gel P-10 of the neutral water-soluble polysaccharides (see Lemassu \& Daffé, 1994). The glycosyl composition is the mean value of three different experiments and is expressed in mol \% of the sugar residues in purified AMs. The amounts of AMs are expressed in $\mu \mathrm{g}$ purified AMs (100 mg bacterial dry wt $)^{-1}$.

\begin{tabular}{|c|c|c|c|c|c|c|c|c|c|c|}
\hline & \multicolumn{10}{|c|}{ Strain } \\
\hline & \multicolumn{2}{|c|}{ Erdman } & \multicolumn{2}{|c|}{ H37Rv } & \multicolumn{2}{|c|}{ H37Ra } & \multicolumn{2}{|c|}{ R1Rv } & \multicolumn{2}{|c|}{$\mathbf{R 1 R a}$} \\
\hline & $\mathbf{E}$ & $\mathbf{S}$ & $\mathbf{E}$ & $\mathbf{S}$ & $\mathbf{E}$ & $\mathbf{S}$ & $\mathbf{E}$ & $\mathbf{s}$ & $\mathbf{E}$ & $\mathbf{S}$ \\
\hline \multicolumn{11}{|c|}{ Glycosyl composition } \\
\hline Ara & $76 \cdot 0$ & $54 \cdot 7$ & $79 \cdot 0$ & $64 \cdot 5$ & $69 \cdot 5$ & $49 \cdot 2$ & $62 \cdot 2$ & $52 \cdot 7$ & $60 \cdot 5$ & $52 \cdot 7$ \\
\hline Man & $24 \cdot 0$ & $45 \cdot 3$ & $21 \cdot 0$ & $35 \cdot 5$ & $30 \cdot 5$ & $50 \cdot 8$ & $37 \cdot 8$ & $47 \cdot 3$ & $39 \cdot 5$ & $47 \cdot 3$ \\
\hline Ara/Man & $3 \cdot 1$ & $1 \cdot 2$ & 3.7 & $1 \cdot 8$ & $2 \cdot 3$ & $1 \cdot 0$ & $1 \cdot 6$ & $1 \cdot 1$ & $1 \cdot 5$ & $1 \cdot 1$ \\
\hline AMs & 16 & 54 & 67 & 88 & 82 & 60 & 8 & 14 & 70 & 35 \\
\hline
\end{tabular}



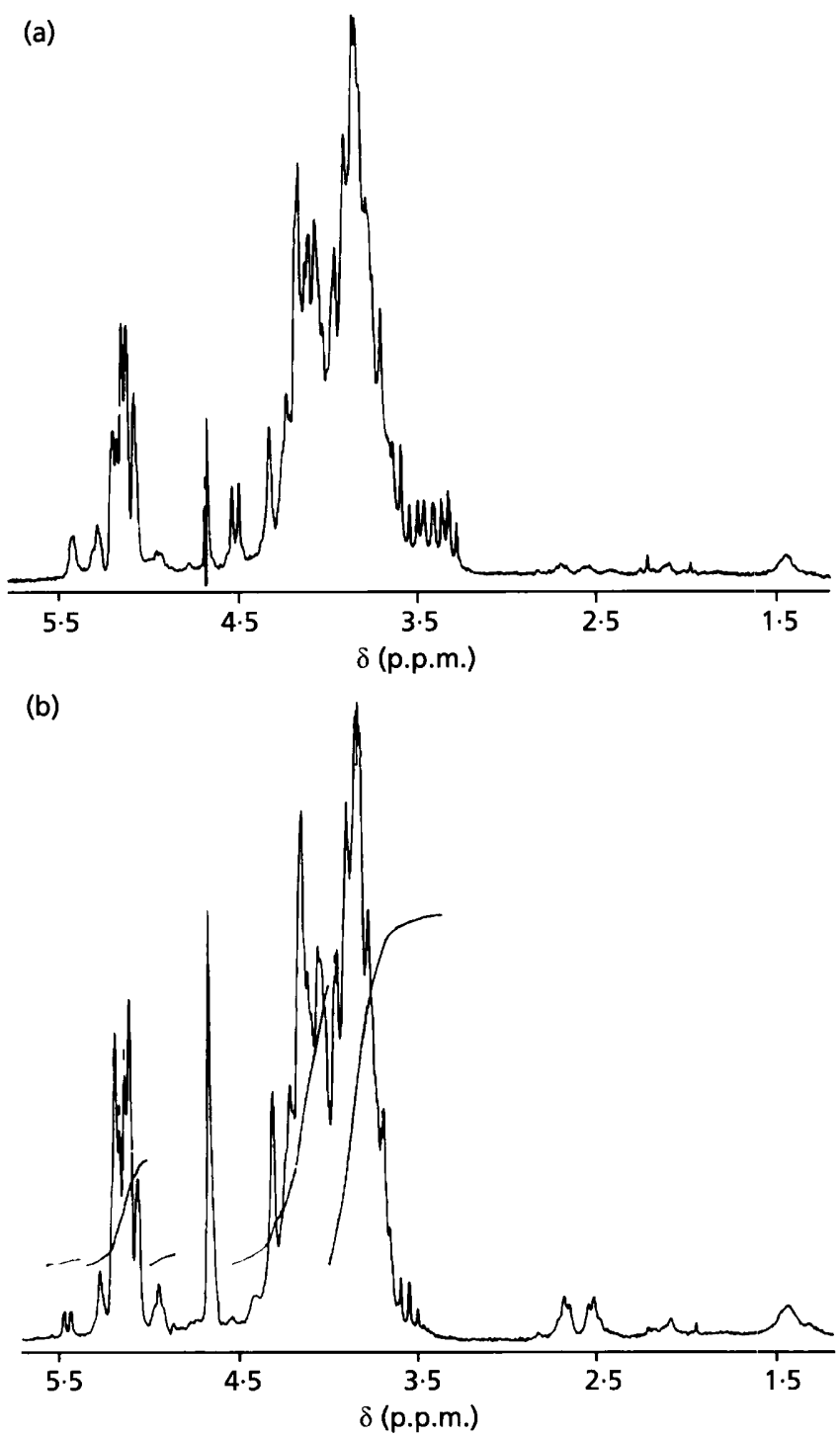

Fig. 1. ${ }^{1} \mathrm{H}-\mathrm{NMR}$ spectra $\left(500 \mathrm{MHz}, 10 \mathrm{mg} \mathrm{D}_{2} \mathrm{O} \mathrm{ml} \mathrm{m}^{-1}\right)$ of (a) neutral and (b) acidic AMs purified from the surface-exposed materials of $M$. tuberculosis (H37Rv). The tracing over the spectrum in (b) represents the integration of proton signals. In both experiments, the HOD signal at 4.7 p.p.m. has been presaturated.

region (0-3 p.p.m.). The failure to detect fatty acyl groups, glycerol and inositol in the acid hydrolysis products of the purified AMs indicates the lack of LAM in these polysaccharide fractions, in agreement with their chromatographic behaviour on the anion-exchange column.

Chromatography of the acidic fractions derived from the DEAE columns of the water-soluble polysaccharides on columns of Bio-Gel P-10 led to the isolation of two compounds: one of them was composed of Ara and Man (Ara/Man $=2 \cdot 2)$ and exhibited the same apparent molecular mass as that of the neutral AMs, and the other peak contained only Xyl. Analysis of the former compound by ${ }^{1} \mathrm{H}-\mathrm{NMR}$ (Fig. 1b) showed the existence of two signals

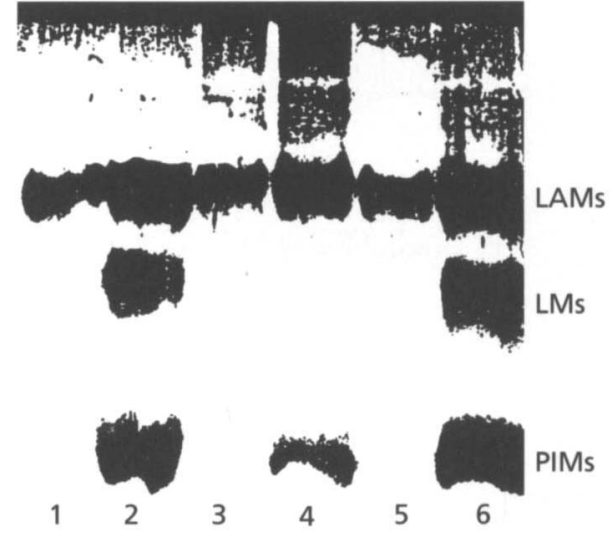

Fig. 2. SDS-PAGE of polysaccharides from $M$. tuberculosis strain H37Rv: $20 \mu \mathrm{g}$ (lane 1) and $50 \mu \mathrm{g}$ (lane 2) water-soluble polysaccharides from the extracellular material; $20 \mu \mathrm{g}$ (lane 3) and $50 \mu \mathrm{g}$ (lane 4) water-soluble polysaccharides from the surface-exposed material; $2 \mu \mathrm{g}$ purified acidic AMs (lane 5); $20 \mu \mathrm{g}$ standard mixtures of LAMs, LMs and PIMs (lane 6).

assignable to the resonances of the two methylene groups of succinyl substituents (Weber \& Gray, 1979) at 2.5 and 2.7 p.p.m. Based on the integration of the anomeric signals (estimated to $93 \mathrm{H}$ for a molecular mass of $13 \mathrm{kDa}$ ), two or three succinyl substituents were present per mole of acidic AM. Furthermore, although neither inositol nor glycerol was detected in this fraction, the compound, but not the neutral AMs, exhibited the same electrophoretic mobility as LAM of $M$. tuberculosis $\mathrm{H} 37 \mathrm{Rv}$ on SDS-PAGE (Fig. 2). The acidic AM was also isolated and characterized from the surface material extracted from $\mathrm{H} 37 \mathrm{Rv}$ by an overnight treatment of the bacilli with Tween 80, thus ruling out the possibility that this compound was derived from LAM by physical shearing. Among the surface-exposed constituents extracted with the detergent or with glass beads, the conventional PIMs were the only phosphomannolipids identified, suggesting that no microbial phospholipase activity (which would generate AMs from LAMs) occurred during the treatments. The acidic AM represents $12-13 \%$ of the watersoluble polysaccharides and roughly $3 \%$ of the surfaceexposed polysaccharides of the tubercle bacillus.

To characterize the reducing end of the neutral AMs, the purified polysaccharides were coupled to the UV-absorbing ethyl $p$-aminobenzoate by reductive amination with cyanoborohydride (Venisse $e t$ al., 1993), hydrolysed and the reducing end products were purified by HPLC. Comparative analysis of these latter compounds and the standard sugar derivatives by GC led to the identification of Man as the sugar terminating the polysaccharides, demonstrating that the polysaccharides differed from LAMs by the absence of a phosphatidylinositol anchor.

\section{Glycosyl-linkage analysis of the major AMs}

In order to determine the linkage and the ring form of the arabinosyl and mannosyl residues, the AMs were per- $O$ methylated, hydrolysed, reduced, acetylated and analysed 
Table 2. Glycosyl-linkage composition and capping of purified AMs from various tubercle bacilli

Purified AMs from extracellular materials (E) and surface-exposed materials (S) were $O$-methylated, hydrolysed, reduced, acetylated and analysed by GC-MS as described by Lemassu \& Daffé (1994). The glycosyl-linkage composition is expressed in mol \% of the different glycosyl residues (data were obtained from three different experiments). The percentage Man-capping is expressed in (mol 3,5Ara - mol t-Ara/mol 3,5-Ara) $\times 100$.

\begin{tabular}{|c|c|c|c|c|c|c|c|c|c|c|}
\hline & \multicolumn{10}{|c|}{ Strain } \\
\hline & \multicolumn{2}{|c|}{ Erdman } & \multicolumn{2}{|c|}{ H37Rv } & \multicolumn{2}{|c|}{ H37Ra } & \multicolumn{2}{|c|}{$\mathbf{R} 1 \mathbf{R v}$} & \multicolumn{2}{|c|}{$\mathbf{R} 1 \mathbf{R a}$} \\
\hline & $\mathbf{E}$ & $\mathbf{S}$ & $\mathbf{E}$ & $\mathbf{S}$ & $\mathbf{E}$ & S & $\mathbf{E}$ & $\mathbf{S}$ & $\mathbf{E}$ & $\mathbf{S}$ \\
\hline \multicolumn{11}{|c|}{ Glycosyl residue } \\
\hline $\mathrm{t}$-Ara & $9 \cdot 5$ & $3 \cdot 5$ & $5 \cdot 5$ & $4 \cdot 0$ & $4 \cdot 0$ & $1 \cdot 0$ & 1.5 & $1 \cdot 5$ & 3.5 & $1 \cdot 5$ \\
\hline 2-Ara & $16 \cdot 0$ & $10 \cdot 2$ & $17 \cdot 0$ & $16 \cdot 5$ & $17 \cdot 0$ & $3 \cdot 5$ & $8 \cdot 0$ & $2 \cdot 5$ & $8 \cdot 3$ & $5 \cdot 0$ \\
\hline 5-Ara & $39 \cdot 0$ & $31 \cdot 5$ & $41 \cdot 5$ & $28 \cdot 0$ & $35 \cdot 5$ & $32 \cdot 5$ & $37 \cdot 0$ & $38 \cdot 5$ & $40 \cdot 5$ & $35 \cdot 0$ \\
\hline 3,5-Ara & $11 \cdot 5$ & $9 \cdot 5$ & $15 \cdot 0$ & $16 \cdot 0$ & $13 \cdot 0$ & $12 \cdot 2$ & $15 \cdot 7$ & $10 \cdot 2$ & $8 \cdot 2$ & $11 \cdot 2$ \\
\hline $\mathrm{t}-\mathrm{Man}$ & $12 \cdot 0$ & $19 \cdot 0$ & $12 \cdot 0$ & $21 \cdot 0$ & $17 \cdot 0$ & $21 \cdot 0$ & $17 \cdot 5$ & $26 \cdot 5$ & $21 \cdot 0$ & 25.5 \\
\hline 2-Man & $6 \cdot 0$ & $11 \cdot 3$ & $4 \cdot 0$ & $10 \cdot 0$ & $6 \cdot 0$ & $11 \cdot 0$ & $11 \cdot 0$ & $8 \cdot 3$ & $9 \cdot 0$ & $10 \cdot 5$ \\
\hline 6-Man & $2 \cdot 0$ & $3 \cdot 5$ & $2 \cdot 0$ & $1 \cdot 0$ & $1 \cdot 5$ & $6 \cdot 5$ & $2 \cdot 8$ & $6 \cdot 0$ & $2 \cdot 0$ & $4 \cdot 0$ \\
\hline 2,6-Man & $4 \cdot 0$ & $11 \cdot 5$ & $3 \cdot 0$ & 3.5 & $6 \cdot 0$ & $12 \cdot 3$ & 6.5 & 6.5 & $7 \cdot 5$ & $7 \cdot 3$ \\
\hline Man-capping & 17 & 63 & 63 & 75 & 69 & 92 & 90 & 85 & 57 & 87 \\
\hline
\end{tabular}

by GC and GC-MS (Table 2). The arabinan segments consisted of terminal (t)-, 2-, 3,5- and 5-linked Araf residues; the presence of substantial amounts of pyranosyl residues was ruled out by NMR analyses (see below). The mannan segments contained $t-, 2-, 6-$ and 2,6-linked pyranosides $(\operatorname{Man} p)$ as all the mannosyl residues, after $O$ methylation, contained a methoxyl group located on C-4, in agreement with the published data (Chatterjee et al., 1991; Lemassu \& Daffé, 1994; Misaki et al., 1977).

Examination of the glycosyl-linkage composition of AraLAM of the rapidly growing unidentified mycobacterium (Chatterjee et al., 1991) and arabinogalactan of M. tuberculosis and other mycobacterial species (Daffé et al., 1993) showed that the number of mol t-A raf corresponded roughly to that of branched 3,5-linked Araf. This was the expected picture for non-capped AMs and LAMs. In contrast, examination of the glycosyl-linkage composition of AMs from the different tubercle bacilli (Table 2) showed a large excess of 3,5-linked Araf units as compared to the small amounts of t-Araf residues. Similarly, the number of $\mathrm{t}-\mathrm{Man} p$ units far exceeded that of 2,6-linked Man $p$ residues. These data supported the existence of mannosyl residues substituting the non-reducing ends of the arabinan segments. The occurrence of appreciable amounts of 2-linked Manp residues in the various AMs (Table 2) also corroborated the phenomenon of capping (Chatterjee et al., 1992a; Lemassu \& Daffé, 1994). The extent of capping of AMs with mannosyl residues may be estimated from the (3,5-linked Araf- $\mathrm{t}$-Araf)/(3,5-linked Araf) ratio which is expected to equal the (t-Man $p-2,6-$ linked Man $p) /(3,5$-linked Araf). The former method of estimation was found to be more accurate than the latter one, probably due to an overestimation of $\mathrm{t}-\mathrm{Man} p$ by the glycosyl-linkage analysis leading sometimes to strong aberrant percentages of capping (Table 2; see also Khoo et al., 1995).

Examination of the percentage of capping of the various AMs, estimated from the (3,5-linked Araf-t-Araf)/(3,5linked Araf) ratio, showed that surface-exposed AMs were generally more capped than the corresponding extracellular molecules. The percentage of capping of the surface-exposed AMs was found to be almost identical for the two R1 strains (Table 2). Surprisingly, the avirulent H37Ra strain contained the most capped AMs, followed by those of the R1 series, H37Rv and Erdman. Among the extracellular AMs, that of R1Rv was the most capped whereas that of the virulent Erdman strain was poorly capped. As capping with mannosyl residues is known to occur via the 2-linked Man $p$-containing oligomannosyl (Chatterjee et al., 1992b; Lemassu \& Daffé, 1994; Venisse et al., 1993), it was interesting to compare the relative amounts of this residue in the various AMs. Analysis of the glycosyl-linkage composition showed that comparable amounts of 2-linked Manp occurred in AMs from the H37 strains, suggesting similar structural features for the polysaccharides (Table 2).

\section{${ }^{13}$ C-NMR analysis of the major AMs}

The ${ }^{13} \mathrm{C}$-NMR spectra of the neutral extracellular and surface-exposed AMs isolated from the various strains were very similar to each other and to those previously published for AMs and deacylated LAMs of M. tuberculosis (Chatterjee et al., 1992b; Lemassu \& Daffé, 1994; Venisse et al., 1993). They differed only in the relative intensity of signals corresponding to the resonances assignable to 


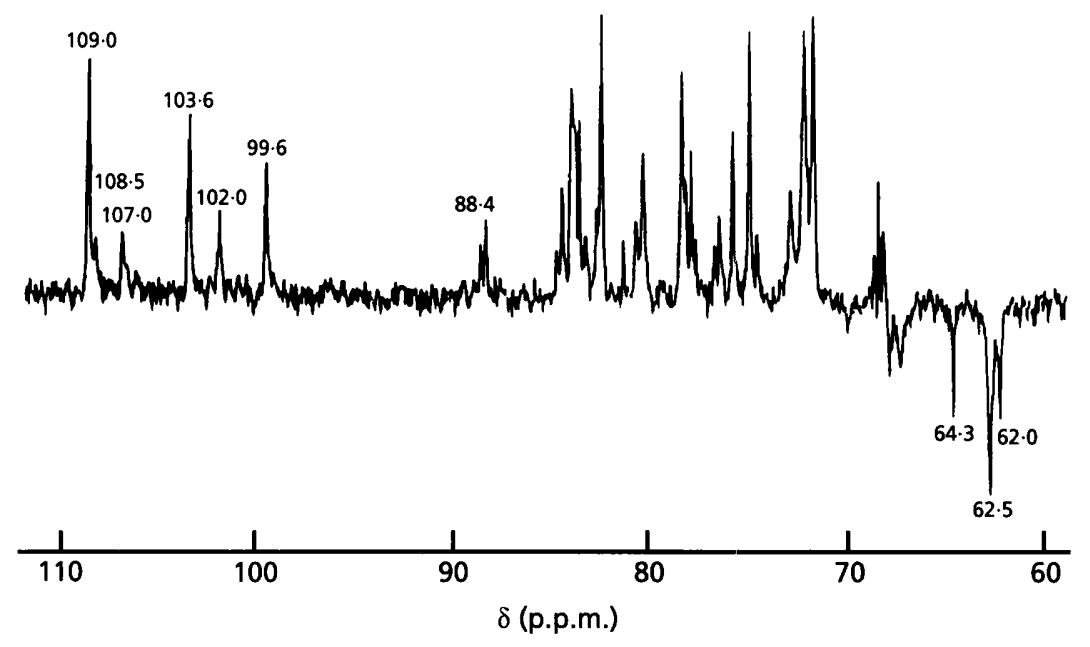

Fig. 3. ${ }^{13} \mathrm{C}-\mathrm{NMR}$ spectrum $(125 \mathrm{MHz}, 25 \mathrm{mg}$ $\mathrm{D}_{2} \mathrm{O} \mathrm{ml}^{-1}$ ) of the neutral AMs purified from the surface-exposed material of $M$. tuberculosis. The spectrum was obtained using a Bruker J-modulated sequence. Thus, the resonance signals of $\mathrm{CH}$ and $\mathrm{CH}_{3}$ were phase positive whereas those of $\mathrm{CH}_{2}$ were phase negative. arabinosyl or mannosyl residues, in agreement with the difference observed in the Ara/Man ratio of the various AMs (Table 1). A representative spectrum is shown in Fig. 3. All the signals were observed between 60 and 110 p.p.m., confirming the lack of fatty acyl substituents on these polysaccharides. It is worth noting that a signal at $64 \cdot 3$ p.p.m., assignable only to the resonance of unsubstituted C- 5 of t $\beta$ - A raf (Bradbury \& Jenkins, 1984; Gorin \& Mazurek, 1975), was observed in all of the ${ }^{13} \mathrm{C}$ NMR spectra, confirming the occurrence of non-capped arabinan segments in the AMs of the various strains (Table 2).

\section{Structure of the mannan core of AMs}

From the glycosyl-linkage analysis, the mannan core of AMs was found to be composed of t-, 2-, 6- and 2,6linked Man $p$ (Table 2) which are known to occur in the $\alpha$ configuration (Lemassu \& Daffé, 1994; Misaki et al., 1977). However, in mycobacterial AMs and LAMs it has been shown that most of the t-Man $p$ and all of the 2-linked Man $p$ were involved in the capping of the termini of the arabinan segment (Chatterjee et al., 1992b; Lemassu \& Daffé, 1994; Venisse et al., 1993). The mannan core of both AMs and LAMs is also known to consist of a $1 \rightarrow 6$ backbone of mannosyl residues substituted with $1 \rightarrow 2$ mannosyl segments (Chatterjee et al., 1992a; Lemassu \& Daffé, 1994; Misaki et al., 1977; Venisse et al., 1993). To determine the length of the oligomannosyl branched on the mannan backbone, AMs were submitted to acetolysis. Analysis of the resulting peracetylated products showed that peracetylated mannoside was the major component of the acetolysis products of all the AMs. It followed then that the different polysaccharides shared a common mannan core.

\section{Characterization of other surface-exposed glycoconjugates}

As the acid hydrolysis products of the organic phases derived from the chloroform/methanol/water partition of the crude polymers also contained Man, it was interesting to identify the mannolipids. Analysis of the organic phases by TLC demonstrated the presence of PIMs in the materials derived from the mechanical treatment of all the strains; no PIM was detected in the organic phases derived from the extracellular materials, in agreement with our previous report showing that the culture filtrates of the tubercle bacilli are devoid of lipid (Lemassu \& Daffé, 1994).

Among the surface-exposed capsular proteins of the tubercle bacillus (Ortalo-Magné et al., 1995), the presence of the two carbohydrate-associated lipoproteins (Young et al., 1992) was also investigated. We compared the amounts of these proteins in tubercle bacilli by immunoblotting using the previously characterized $\mathrm{mA}$ bs HYT 6 and HBT 12 directed against the 19 and the $38 \mathrm{kDa}$ lipoprotein, respectively (Andersen et al., 1986; Ljungqvist $e$ al., 1988). As shown in Fig. 4, the avirulent R1Ra strain and the virulent H37Rv strain contain comparable amounts of 19 and $38 \mathrm{kDa}$ glycoproteins, both as secreted and surface-exposed proteins. The attenuated strain R1Rv contained much less amounts of lipoproteins than did the avirulent $\mathrm{R} 1 \mathrm{Ra}$ strain. These data demonstrated that no obvious correlation exists in the mannose-associated lipoprotein content and the degrees of virulence of the examined strains.

\section{Chemical analyses of fatty acids of whole cells}

Tubercle bacilli are well known to exhibit various degrees of virulence for guinea pigs (Steenken \& Gardner, $1946 \mathrm{a}, \mathrm{b})$ and previous studies suggested a correlation between the occurrence of $\alpha, \beta$-unsaturated trimethylbranched fatty acids (phthienoic acids) and virulence of tubercle bacilli (Asselineau, 1982; Daffé et al., 1983). Among the strains used in the present study, the H37 strains were the only strains examined for their content in these fatty acids (Daffé $e t$ al., 1983). Thus, we extended the chemical analysis of fatty acid content to the three other strains in order to ensure that all the strains used for isolating the arabinomannan-containing glycans belong to the M. tuberculosis complex and to check the validity of the proposed correlation. Three different types of mycolic 
(a)

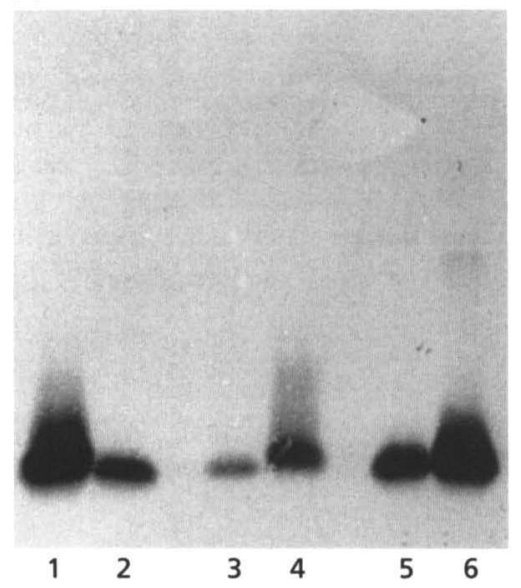

(b)

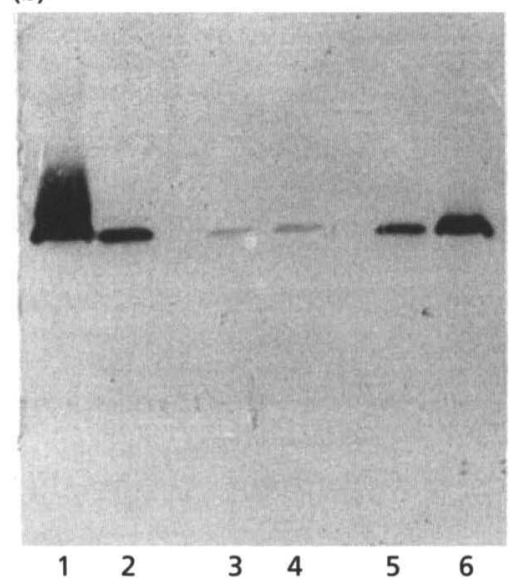

Fig. 4. Immunoblots onto nitrocellulose of the culture filtrate (lanes 2, 3 and 5) and surface-exposed materials (lanes 1, 4 and 6) of $M$. tuberculosis H37Rv (lanes 1 and 2), R1Rv (lanes 3 and 4) and R1Ra (lanes 5 and 6) probed with (a) HYT 6 (anti-19-kDa lipoprotein) and (b) HBT12 (anti-38-kDa lipoprotein).

acids ( $\alpha$-branched, $\beta$-hydroxylated $\mathrm{C} 60-\mathrm{C} 90$ fatty acids) were detected in all the strains examined, namely dicyclopropanic, keto- and methoxylated mycolic acids. The main pyrolysis product identified from these compounds was hexacosanoic acid, demonstrating that all the strains belonged to the M. tuberculosis complex (Daffé et al., 1983). GC analysis of the fatty methyl esters showed the presence of the characteristic multi-methyl-branched long chains (mycocerosic acids) in all the strains and of phthienoic acids in the virulent strains Erdman and H37Rv and not in the attenuated strain $\mathrm{R} 1 \mathrm{Rv}$ and the avirulent strains $\mathrm{H} 37 \mathrm{Ra}$ and R1Ra. These data clearly confirmed the identification of the strains as $M$. tuberculosis and are in agreement with the previously suggested correlation between the occurrence of phthienoic acids and the virulence of the tubercle bacilli.

\section{DISCUSSION}

Among the key matters to address for elucidating the molecular basis of the pathogenesis of tuberculosis is the interaction between the pathogen and phagocytic cells. The outermost surface components of the tubercle bacillus are necessarily involved in this interaction. Interestingly, it has been recently shown that serum components play important roles in mediating phagocytosis of both virulent and avirulent tubercle bacilli but that the mannose receptors also play an important role in phagocytosis of virulent strains (Schlesinger, 1993). Indeed, entry via a specific pathway may provide virulent tubercle bacilli safe passage into phagocytes by allowing them to avoid the toxic consequences of the oxidative burst as previously postulated by Schlesinger $e t$ al. (1990). However, the presence of ManLAMs in virulent and avirulent tubercle bacilli (Khoo et al., 1995) suggested that these molecules are not the likely candidates to mediate the selective interaction of virulent bacilli with the mannose receptors of the phagocytes. Consequently, in the present study, we addressed the question of the chemical nature of the mannose receptor ligand(s) by purifying and chemically characterizing the outermost capsular mannose-containing glycans of five strains of $M$. tuberculosis differing by their degrees of virulence. It appeared that (i) the outermost glycans of virulent and avirulent tubercle bacilli contained no detectable amount of LAM, clearly demonstrating that LAMs are not involved in the interaction of the tubercle bacillus with the mannose receptors of phagocytes. Instead, the outermost capsular polysaccharides were composed mostly of neutral AMs and small amounts of acidic polysaccharides; (ii) AMs are heterogeneous, both in terms of glycosyl composition (Ara/Man ratio) and of capping with mannosyl residues; (iii) no obvious difference exists between the capping of AMs of virulent and avirulent tubercle bacilli. This latter result is consistent with the recent demonstration of similar capping of the structurally related LAMs from avirulent and virulent strains of tubercle bacilli (Khoo et al., 1995). Besides, the immunochemical data on the surface location of LAMs (Hunter \& Brennan, 1990) may now be re-interpreted, in view of the data presented herein, as demonstrating the exposure of AMs on the cell surface.

The possibility that the mannosyl residues of ManAMs are surface-expressed in virulent tubercle bacilli and not in the avirulent ones also exists. We tested this hypothesis by using L9 $\mathrm{mAb}$ directed against the arabinan segments which reacted with the cell surface of $M$. tuberculosis (Ortalo-Magné et al., 1995) and L4 mAb whose epitope has been delineated as involving the axial 2-hydroxyl of $\alpha$-D-mannosyl residues (Fournié et al., 1991). Using cytofluorimetry, we showed that the arabinan segments, as well as the mannosyl residues, were surface-exposed in virulent and avirulent strains tested but failed to correlate the extent of exposure of these residues and the virulence of the strains (A. Ortalo-Magné, V. Ozanne, A. Vercelonne, J. J. Fournié \& M. Daffé, unpublished results).

Another possibility may reside in the surface exposure in virulent strains of other glycoconjugates. This led us to investigate the implication of two other classes of previously known Man-containing glycoconjugates, 
namely PIMs (Goren \& Brennan, 1979) and Manassociated glycoproteins (Espitia et al., 1993, 1995). Although PIMs are partly surface-exposed, only a tiny amount of these compounds are present on the cell surface, since lipids represent at best $6 \%$ of the surfaceexposed material (Ortalo-Magné et al., 1995). In addition, PIMs are not good candidates to mediate the interaction with the mannose receptor, as they share with LMs the same structural features (Chatterjee et al., 1992a) which have been shown not to mediate the interaction with phagocytes (Schlesinger et al., 1994). Similarly, the two Man-associated lipoproteins so far characterized in the tubercle bacilli, i.e. the 19 and $38 \mathrm{kDa}$ lipoproteins (Young et al., 1992), are present in the outermost compartment of the capsule of both virulent and avirulent tubercle bacilli in comparable amounts.

It is also possible to postulate a high specificity of the interaction between the mannose receptors and the Mancontaining surface-exposed bacterial components and the existence of subtle structural differences between the AMs of virulent and avirulent strains. In connection with this hypothesis, it is interesting to note that although the LAMs of bacillus Calmette-Guérin (BCG) are as capped as those of virulent strains of tubercle bacilli (Khoo et al., 1995; Venisse et al., 1993), ManLAMs of BCG contain several mannosyl segments not previously characterized in the virulent strain H37Rv (Prinzis et al., 1994). Further studies are clearly needed in order to decipher the chernical basis of the interaction between the tubercle bacillus and the macrophage.

The lack of correlation between the degrees of virulence of tubercle bacilli and their content in mannoconjugates examined herein, the most likely candidates to mediate the interaction between the tubercle bacillus and the mannose receptors of phagocytes, prompts further investigation using more strains of avirulent tubercle bacilli, pathogenic and non-pathogenic mycobacterial species.

Finally, the neutral AMs characterized from the cell surface components of the tubercle bacilli may function as virulence factors in the pathogenesis of mycobacterial diseases, since AMs of $M$. tuberculosis have been shown to suppress macrophage-dependent, antigen-induced activation of human lymphocytes (Ellner \& Daniel, 1979) and to inhibit the processing of antigen by antigenpresenting T-cells (Moreno et al., 1988), two important functions of host cell-mediated immunity. Besides, the presence of small amounts of an acidic AM exhibiting the same mobility on SDS-PAGE as that of LAM urges further comparison of the structures and the biologic activities of the two molecules.

\section{ACKNOWLEDGEMENTS}

We are grateful to Dr P. Draper (NIMR, London), Professor G. Lanéelle and Dr M.-A. Lanéelle (both from Université Paul Sabatier and LPTF-CNRS, Toulouse) for stimulating discussions. We also thank Drs H. Montrozier and J. Roussel, and Mrs V. Ozanne and T. Brando (LPTF-CNRS, Toulouse) for their excellent technical help. This work was supported in part by contract no. 920609 from the Institut National de la Santé et de la Recherche Médicale (INSERM, France).

\section{REFERENCES}

Andersen, Å. B., Yuan, Z. L., Haslov, K., Vergmann, B. \& Bennedsen, J. (1986). Interspecies reactivity of five monoclonal antibodies to Mycobacterium tuberculosis as examined by immunoblotting and enzyme-linked immunosorbent assay. Infect Immun 23, 446-451.

Asselineau, J. (1982). Branched-chain fatty acids of mycobacteria. Indian J Chest Dis 24, 143-157.

Bermudez, L. E., Young, L. S. \& Enkel, H. (1991). Interaction of Mycobacterium avium complex with human macrophages: roles of membrane receptors and serum proteins. Infect Immun 59, $1697-1702$.

Bloom, B. R. \& Murray, J. L. (1992). Tuberculosis : commentary on a reemergent killer. Science 257, 1055-1064.

Bradbury, J. H. \& Jenkins, G. A. (1984). Determination of the structures of trisaccharides by ${ }^{13} \mathrm{C}-\mathrm{NMR}$ spectroscopy. Carbobydr Res 126, 125-156.

Chatterjee, D., Bozic, C., McNeil, M. \& Brennan, P. J. (1991). Structural features of the arabinan component of the lipoarabinomannan of Mycobacterium tuberculosis. J Biol Chem 266, $9652-9660$

Chatterjee, D., Hunter, S. W., McNeil, M. \& Brennan, P. J. (1992a). Lipoarabinomannan. Multiglycosylated form of the mycobacterial mannosylphosphatidylinositols. J Biol Chem 267, 6228-6233.

Chatterjee, D., Lowell, K., Rivoire, B., McNeil, M. R. \& Brennan, P. J. (1992b). Lipoarabinomannan of Mycobacterium tuberculosis: capping with mannosyl residues in some strains. $J$ Biol Chem 267, 6234-6239.

Daffé, M., Lanéelle, M.-A., Asselineau, C., Lévy-Frébault, v. \& David, H. L. (1983). Intérêt taxonomique des acides gras des mycobactéries: proposition d'une méthode d'analyse. Ann Microbiol Inst Pasteur 134, 367-377.

Daffé, M., Brennan, P. J. \& McNeil, M. (1990). Predominant structural features of the cell wall arabinogalactan of Mycobacterium tuberculosis as revealed through characterization of oligoglycosyl alditol fragments by gas chromatography/mass spectrometry and by ${ }^{1} \mathrm{H}$ - and ${ }^{13} \mathrm{C}-\mathrm{NMR}$ analyses. $J$ Biol Chem 265, 6734-6743.

Daffé, M., Brennan, P. J. \& McNeil, M. (1993). Major structural features of the cell wall arabinogalactans of Mycobacterium, Rhodococcus, and Nocardia spp. Carbobydr Res 249, 383-398.

Dittmer, J. C. F. \& Lester, R. L. (1964). A simple specific spray for the detection of phospholipids on thin layer chromatograms. $J$ Lipid Res 5, 126-127.

Ellner, J. J. \& Daniel, T. M. (1979). Immunosuppression by mycobacterial arabinomannan. Clin Exp Immunol 35, 250-257.

Espitia, C. \& Mancilla, R. (1989). Identification, isolation and partial characterization of Mycobacterium tuberculosis glycoprotein antigens. Clin Exp Immunol 77, 378-383.

Espitia, C., Onate, A., Zhang, Y., Young, D. \& Moreno, C. (1993). Biochemical characterization of native and recombinant Con-A binding proteins of Mycobacterium tuberculosis. Abstr Second Int Conf Patbog Mycobact Infect (Stockholm), 52.

Espitia, C., Espinosa, R., Saavedra, R., Mancilla, R., Romain, F., Laqueyrerie, A. \& Moreno, C. (1995). Antigenic and structural similarities between Mycobacterium tuberculosis 50- to 55-kilodalton and Mycobacterium bovis BCG 45- to 47-kilodalton antigens. Infect Immun 63, 580-584.

Fournié, J.-J., Mullins, R. J. \& Basten, A. (1991). Isolation and 
structural characteristics of a monoclonal antibody-defined crossreactive phospholipid antigen from Mycobacterium tuberculosis and Mycobacterium leprae. J Biol Chem 266, 1211-1219.

Garbe, T., Harris, D., Vordermeier, M., Lathigra, R., Ivanyi, J. \& Young, D. (1993). Expression of the Mycobacterium tuberculosis 19 kilodalton antigen in Mycobacterium smegmatis: immunological analysis and evidence of glycosylation. Infect Immun 61, 260-267.

Goren, M. B. \& Brennan, P. J. (1979). Mycobacterial lipids. In Tuberculosis, pp. 69-193. Edited by G. P. Youmans. Philadelphia: W. B. Saunders.

Gorin, A. J. \& Mazurek, M. (1975). Further studies on the assignment of signals in ${ }^{13} \mathrm{C}$ magnetic resonance spectra of aldoses and derived methyl glycosides. Can J Chem 53, 1212-1223.

Hetland, G. \& Wiker, H. G. (1994). Antigen $85 C$ on Mycobacterium bovis BCG and $M$. tuberculosis promotes monocyte-CR3-mediated uptake of microbeads coated with mycobacterial products. Immuno$\log y$ 82, 445-449.

Hirsch, C. S., Ellner, J. J., Russell, D. G. \& Rich, E. A. (1994). Complement receptor-mediated uptake and tumor necrosis factor$\alpha$-mediated growth inhibition of Mycobacterium tuberculosis by human alveolar macrophages. J Immunol 152, 743-753.

Hunter, S. W. \& Brennan, P. J. (1990). Evidence for the presence of a phosphatidylinositol anchor on the lipoarabinomannan and lipomannan of Mycobacterium tuberculosis. J Biol Chem 265, 9272-9279.

Khoo, K.-H., Dell, A., Morris, H. R., Brennan, P. J. \& Chatterjee, D. (1995). Inositol phosphate capping of the nonreducing termini of lipoarabinomannan from rapidly growing strains of Mycobacterium. $J$ Biol Cbem 270, 12380-12389.

Kochi, A. (1991). The global tuberculosis situation and the new control strategy of the World Health Organization. Tubercle 72, $1-6$.

Lemassu, A. \& Daffé, M. (1994) Structural features of the exocellular polysaccharides of Mycobacterium tuberculosis. Biocbem J 297, 351-357.

Ljungqvist, L., Worsaae, A. \& Heron, I. (1988). Antibody responses against Mycobacterium tuberculosis in 11 strains of inbred mice: novel monoclonal antibody specificities generated by fusions, using spleens from BALB.B10 and CBA/J mice. Infect Immun 56, 1994-1998.

Misaki, A., Azuma, I. \& Yamamura, Y. (1977). Structural and immunochemical studies on D-arabino-D-mannans and D-mannans of Mycobacterium tuberculosis and other Mycobacterium species. J Biochem 82, 1759-1770.

Moreno, C., Mehlert, A. \& Lamb, J. (1988). The inhibitory effects of mycobacterial lipoarabinomannan and polysaccharides upon polyclonal and monoclonal human $\mathrm{T}$ cell proliferation. Clin Exp Immunol 74, 206-210.

Ortalo-Magné, A., Dupont, M.-A., Lemassu, A., Andersen, A. B., Gounon, P. \& Daffé, M. (1995). Molecular composition of the outermost capsular material of the tubercle bacillus. Microbiology 141, 1605-1620.

Prinzis, S., Chatterjee, D. \& Brennan, P. J. (1994). Structure and antigenicity of lipoarabinomannan from Mycobacterium bovis BCG. $J$ Gen Microbiol 139, 2649-2658.

Sauton, B. (1912). Sur la nutrition minérale du bacille tuberculeux. C R Acad Sci Ser III Sci Vie 155, 860-863.

Schlesinger, L. S. (1993). Macrophage phagocytosis of virulent but not attenuated strains of Mycobacterium tuberculosis is mediated by mannose receptors in addition to complement receptors. $J$ Immunol 150, 2920-2930.

Schlesinger, L. S. \& Horwitz, M. A. (1990). Phagocytosis of leprosy bacilli is mediated by complement receptors CR1 and CR3 on human monocytes and complement component $\mathrm{C} 3$ in serum. J Clin Invest 85, 1304-1314.

Schlesinger, L. S. \& Horwitz, M. A. (1991). Phagocytosis of Mycobacterium leprae by human monocyte-derived macrophages is mediated by complement receptors CR1 (CD35), CR3 (CD11b/CD18), CR4 (CD11c/CD18) and IFN- $\gamma$ activation inhibits complement receptor function and phagocytosis of this bacterium. J Immunol 147, 1983-1994.

Schlesinger, L. S., Bellinger-Kawahara, C. G., Payne, N. R. \& Horwitz, M. A. (1990). Phagocytosis of Mycobacterium tuberculosis is mediated by human monocyte complement receptors and complement component C3. J Immunol 144, 2771-2780.

Schlesinger, L. S., Hull, S. R. \& Kaufman, T. M. (1994). Binding of the terminal mannosyl units of lipoarabinomannan from a virulent strain of Mycobacterium tuberculosis to human macrophages. J Immunol 152, 4070-4079.

Steenken, W. \& Gardner, L. U. (1946a). R1 strain of tubercle bacillus. $A m$ Rev Tuberc 54, 51-61.

Steenken, W. \& Gardner, L. U. (1946b). History of H37 strain of tubercle bacillus. Am Rev Tuberc 54, 62-66.

Stokes, R. W., Haidl, I. D., Jefferies, W. A. \& Speert, D. P. (1993). Mycobacteria-macrophage interactions. Macrophage phenotype determines the nonopsonic binding of Mycobacterium tuberculosis to murine macrophages. J Immunol 151, 7067-7076.

Venisse, A., Berjeaud, J.-M., Chaurand, P., Gilleron, M. \& Puzo, G. (1993). Structural features of lipoarabinomannan from $M y c o-$ bacterium bovis BCG. J Biol Chem 268, 12401-12411.

Weber, P. L. \& Gray, G. R. (1979). Structural and immunochemical characterization of the acidic arabinomannan of Mycobacterium smegmatis. Carbohydr Res 74, 259-278.

Young, D. B., Kaufmann, S. H. E., Hermans, P. W. M. \& Thole, J. E. R. (1992). Mycobacterial protein antigens: a compilation. Mol Microbiol 6, 133-145.

Received 14 September 1995; revised 13 November 1995; accepted 28 November 1995. 